\title{
CT Angiography in Evaluation of Peripheral Vascular Disease and Comparison with Color Doppler Ultrasound
}

\author{
Deepa S Benegal ${ }^{1}$, Chandrashekar H M ${ }^{2}$, Naveen J ${ }^{3}$, Vedaraju ${ }^{4}$ \\ ${ }^{1}$ Senior Resident, Department of Radiodiagnosis, Bangalore Medical College and Research Institute, Bangalore, ${ }^{2}$ Assistant \\ Professor, Department of Radiodiagnosis, Bangalore Medical College and Research Institute, Bangalore, ${ }^{3}$ Assistant Professor, \\ Department of Radiodiagnosis, Bangalore Medical College and Research Institute, Bangalore, ${ }^{4}$ Professor, Department of \\ Radiodiagnosis, Bangalore Medical College and Research Institute, Bangalore, India
}

Corresponding author: Dr Chandrashekar H M, Assistant Professor, Department of Radiodiagnosis, Bangalore Medical College and Research Institute, Bangalore, Victoria Hospital, BMC\&RI, Bengaluru-02, India

DOI: http://dx.doi.org/10.21276/ijcmsr.2020.5.1.55

How to cite this article: Deepa S Benegal, Chandrashekar H M, Naveen J, Vedaraju. CT angiography in evaluation of peripheral vascular disease and comparison with color doppler ultrasound. International Journal of Contemporary Medicine Surgery and Radiology. 2020;5(1):A251-A255.

\section{A B S T R A C T}

Introduction: Peripheral arterial disease is the narrowing or occlusion of arteries of the limb, resulting in complications ranging from minor disabilities to loss of the limb. Colour Doppler ultrasonography has been used as the initial imaging modality followed by Computed tomographic angiography in symptomatic patients. Present study evaluated the comparison between these two modalities in patients with peripheral vascular disease.

Material and methods: A total of 100 patients above 18 years of age with peripheral vascular disease were included in our study. After taking an informed consent a brief history was taken regarding symptomatology, risk factors and duration of disease. Patients were studied by using both Colour Doppler ultrasonography and Computed tomographic angiography to interpret lower limb arterial system and the comparison was made between the two modalities.

Results: Our study shows consistent association of risk factors such as increasing age, smoking, hypertension and diabetic mellitus with the disease. Early stenotic lesions were better picked by ultrasound. In infrapopliteal segments CT angiography was able to detect $20 \%$ positive cases with grade $3 \& 4$ stenosis in comparison to the Ultrasound which was able to detect $12 \%$. Vessel calcifications and collaterals were better detected by computed tomographic angiography.

Conclusion: In assessing patients with mild disease who need medical therapy, ultrasound is the better modality. In patients with moderate to severe disease in whom surgical intervention is required, computed tomographic angiography is preferred for thorough evaluation of entire lower limb arterial tree.

Keywords: Peripheral Arterial Disease, Colour Doppler Ultrasound, Computed Tomographic Angiography, Stenosis.

\section{INTRODUCTION}

Peripheral arterial occlusive disease (PAD) is the narrowing or occlusion of an artery or arteries of the limb. Most commonly caused by atherosclerosis, less common causes include thromboembolism, trauma, entrapment syndromes and vasculitis including vasospastic disorders and buerger's disease. ${ }^{1}$ Disorder of the arterial system produces complications ranging from minor disabilities to loss of the limb. Therefore, early diagnosis and effective treatment is usually rewarded with good outcome and averts dreadful complications.

Conventional digital subtraction angiography is considered as the gold-standard technique. There are several alternative imaging modalities to DSA, including Duplex ultrasonography (DUS), computed tomography angiography (CTA) and magnetic resonance angiography (MRA). ${ }^{2}$

DUS has been used as the initial imaging modality in mild symptomatic PAD. It is operator dependant, time consuming and is less accurate in assessing distal vasculature and collaterals. ${ }^{3}$ With advancement in CT angiography, especially in the MDCT, larger body volumes can be scanned within short time period at high resolution to provide good delineation of arterial flow. This has enabled MDCT to become a promising new, fast, accurate, safe and non invasive modality in lower extremity arterial imaging for diagnosing, for grading, for potential usefulness and type of treatment. ${ }^{4}$ Present study has been conducted with the objective of evaluating and comparing the efficacy of MDCT angiography and color Doppler ultrasonography in diagnosing and assessing severity of peripheral vascular disease.

\section{MATERIAL AND METHODS}

The main source of data was patients presenting to Victoria Hospital and Bowring \& Lady Curzon Hospital attached to Bangalore Medical College and Research Institute, Bangalore during the time period of November 2016 to may 2018 with suspected peripheral vascular disease on clinical examination including claudication, rest pain and tissue loss. After taking 
an informed written consent, a brief history was taken from each patient regarding symptomatology, risk factors and duration of disease. A focused clinical examination with the aid of the referring physician was undertaken as regards to severity and extent of involvement of the disease. A total of 100 patients were included in our study. Patients with history of trauma, altered renal function tests and history of contrast allergy were excluded in our study.

CDUS: Patients were scanned under Philips Affinity G machine in the supine position. Curvilinear $3.5 \mathrm{MHz}$ probe was used to examine the aorta, common, and external iliac arteries. Linear 7.5 MHz probe was used to examine superficial femoral, deep femoral, popliteal, tibioperoneal trunk, posterior tibial, anterior tibial and peroneal arteries.

CT Peripheral Angiography: Patients underwent MDCTA on a "Ingenuity" 128 slice Philps CT scanner. Plain CT Acquisition is done from the level of infrarenal aorta till the toes. Arterial phase was taken when the ROI reaches threshold of $100 \mathrm{HU}$ in descending infrarenal aorta following contrast injection. Venous phase was taken at an interval of 60-65 sec from the time of contrast injection. 3D reconstruction with thin planar $(1 \mathrm{~mm}) \mathrm{MPR}$ was performed in coronal and sagittal planes. The images were viewed on a workstation capable of viewing source axial images and 3D imaging software tools.

\section{Data analysis}

The lower limb arterial system was divided into 3 anatomical regions [ Aorto-iliac region, Femoro-popliteal region \& Tibioperoneal regions ] and 11 anatomical segments [ Infrarenal aorta, common iliac arteries, external iliac arteries, common femoral arteries, Superficial femoral artery, Deep femoral artery, popliteal artery, tibioperoneal trunk, anterior tibial artery, posterior tibial artery, peroneal artery]. Each and every anatomical segment of the arterial tree was assigned a grade for the disease extent using a five point ordinal scale: Grade 0- Normal, Grade 1- < 25\% diameter reduction, Grade 2- 25-50\% diameter reduction, Grade 3- 50-75\% diameter reduction, Grade 4- 75-100\% diameter reduction. These grades were given for all arterial segments in both the techniques. MDCT angiography and Doppler findings were compared for each arterial segment. For all practical purpose to analyse the data we had divided the grades of stenosis into hemodynamically significant [Grade $3 \& 4$ ] and hemodynamically nonsignificant [Grade 0,1 \& 2].

\section{STATISTICAL ANALYSIS}

Descriptive and inferential statistical analysis has been carried out in the present study. Results on continuous measurements are presented on Mean \pm SD (Min-Max) and results on categorical measurements are presented in Number (\%). Significance is assessed at 5\% level of significance. Chi-square/ Fisher Exact test has been used to find the significance of study parameters on categorical scale between two or more groups and non-parametric setting used for Qualitative data analysis. Fisher Exact test used when cell samples are very small. The Statistical software namely SPSS 18.0, and $\mathrm{R}$ environment ver.3.2.2 were used for the analysis of the data and Microsoft word and Excel have been used to generate graphs, tables etc.

\section{RESULTS}

A total of 100 patients were included in our study. Most patients belonged to the age group of 40-70 years with half of the patients were smokers, diabetics and hypertensives. Out of 58 patients with significant stenosis, $65.5 \%$ cases were smokers and $24.1 \%$ were non smokers, $55.2 \%$ cases were hypertensives and $24.1 \%$ were non hypertensives, $79.3 \%$ cases were diabetics and $20.7 \%$ were non diabetics, showing significant statistical correlation of smoking, hypertension \& diabetes with significant stenosis.

Out of 300 vessel segments in aortoiliac region 238(79.3\%), 28(9.3), 10(3.3\%), 12(4\%), 12(4\%) segments on CTA and 218(72.7\%), 44(14.7\%), 14(4.7\%), 12(4\%), 12(4\%) segments on CDUS showed grade $0,1,2,3,4$ stenosis respectively (table-1).

Out of 400 vessel segments in tibioperoneal region 288 (72\%), 14 (3.5\%), 22 (5.5\%), 44 (11\%), 36 (9\%) segments

\begin{tabular}{|c|c|c|c|c|c|}
\hline \multicolumn{6}{|c|}{ Stenosis Severity Grade } \\
\hline Modality & 0 & 1 & 2 & 3 & 4 \\
\hline & \multicolumn{5}{|c|}{ Infrarenal aorta segments } \\
\hline CTA & 82 & 16 & 0 & 0 & 2 \\
\hline \multirow[t]{2}{*}{ CDUS } & 76 & 20 & 2 & 0 & 2 \\
\hline & \multicolumn{5}{|c|}{ Common iliac artery segments } \\
\hline CTA & 82 & 4 & 2 & 8 & 4 \\
\hline \multirow[t]{2}{*}{ CDUS } & 76 & 8 & 4 & 8 & 4 \\
\hline & \multicolumn{5}{|c|}{ External iliac segments } \\
\hline CTA & 74 & 8 & 8 & 4 & 6 \\
\hline \multirow[t]{2}{*}{ CDUS } & 66 & 16 & 8 & 4 & 6 \\
\hline & \multicolumn{5}{|c|}{ Common femoral segments } \\
\hline CTA & 66 & 6 & 20 & 2 & 6 \\
\hline \multirow[t]{2}{*}{ CDUS } & 62 & 10 & 20 & 4 & 4 \\
\hline & \multicolumn{5}{|c|}{ Superficial femoral segments } \\
\hline CTA & 54 & 8 & 2 & 8 & 28 \\
\hline \multirow[t]{2}{*}{ CDUS } & 48 & 10 & 8 & 6 & 28 \\
\hline & \multicolumn{5}{|c|}{ Deep femoral segments } \\
\hline CTA & 96 & 4 & 0 & 0 & 0 \\
\hline \multirow[t]{2}{*}{ CDUS } & 82 & 16 & 2 & 0 & 0 \\
\hline & \multicolumn{5}{|c|}{ Popliteal segments } \\
\hline CTA & 80 & 4 & 6 & 6 & 4 \\
\hline \multirow[t]{2}{*}{ CDUS } & 72 & 12 & 8 & 4 & 4 \\
\hline & \multicolumn{5}{|c|}{ Tibioperoneal trunk segments } \\
\hline CTA & 74 & 4 & 6 & 10 & 6 \\
\hline \multirow[t]{2}{*}{ CDUS } & 70 & 12 & 10 & 4 & 4 \\
\hline & \multicolumn{5}{|c|}{ Posterior tibial segments } \\
\hline CTA & 66 & 4 & 4 & 12 & 14 \\
\hline \multirow[t]{2}{*}{ CDUS (96\%) } & 64 & 10 & 12 & 8 & 6 \\
\hline & \multicolumn{5}{|c|}{ Anterior tibial segments } \\
\hline CTA & 68 & 4 & 6 & 14 & 8 \\
\hline \multirow[t]{2}{*}{ CDUS (96\%) } & 68 & 8 & 10 & 12 & 2 \\
\hline & \multicolumn{5}{|c|}{ Peroneal segments } \\
\hline CTA & 74 & 2 & 6 & 8 & 10 \\
\hline CDUS (96\%) & 72 & 8 & 8 & 8 & 4 \\
\hline $\begin{array}{l}\text { Table-1: Co } \\
\text { CDUS of }\end{array}$ & seg & $\begin{array}{l}\text { noro } \\
\text { sin }\end{array}$ & 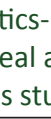 & is 1 & and \\
\hline
\end{tabular}




\begin{tabular}{|c|c|c|c|c|c|}
\hline \multicolumn{6}{|c|}{ Stenosis Severity Grade } \\
\hline Modality & 0 & 1 & 2 & 3 & 4 \\
\hline & \multicolumn{5}{|c|}{ Tibioperoneal trunk segments } \\
\hline CTA & 74 & 4 & 6 & 10 & 6 \\
\hline CDUS & 70 & 12 & 10 & 4 & 4 \\
\hline \multicolumn{6}{|c|}{ Posterior tibial segments } \\
\hline CTA & 66 & 4 & 4 & 12 & 14 \\
\hline CDUS (96\%) & 64 & 10 & 12 & 8 & 6 \\
\hline \multicolumn{6}{|c|}{ Anterior tibial segments } \\
\hline CTA & 68 & 4 & 6 & 14 & 8 \\
\hline CDUS (96\%) & 68 & 8 & 10 & 12 & 2 \\
\hline \multicolumn{6}{|c|}{ Peroneal segments } \\
\hline CTA & 74 & 2 & 6 & 8 & 10 \\
\hline CDUS (96\%) & 72 & 8 & 8 & 8 & 4 \\
\hline
\end{tabular}

\begin{tabular}{|c|c|c|c|c|c|c|c|}
\hline \multicolumn{6}{|c|}{ Stenosis Severity Grade } & \multirow{2}{*}{$\begin{array}{l}\text { Total number } \\
\text { of segments }\end{array}$} & \multirow[t]{2}{*}{$P$ value } \\
\hline Modality & 0 & 1 & 2 & 3 & 4 & & \\
\hline & \multicolumn{5}{|c|}{ Aortoiliac segments } & & \\
\hline CTA & $238(79.3 \%)$ & $28(9.3 \%)$ & $10(3.3 \%)$ & $12(4.0 \%)$ & $12(4.0 \%)$ & 300 & \multirow[t]{2}{*}{0.277} \\
\hline \multirow[t]{2}{*}{ CDUS } & $218(72.7 \%)$ & $44(14.7 \%)$ & $14(4.7 \%)$ & $12(4.0 \%)$ & $12(4.0 \%)$ & 300 & \\
\hline & \multicolumn{5}{|c|}{ Femoropopliteal segments } & & \\
\hline CTA & $296(74 \%)$ & $22(5.5 \%)$ & $28(7 \%)$ & $16(4 \%)$ & $38(9.5 \%)$ & 400 & \multirow[t]{2}{*}{$0.022^{*}$} \\
\hline \multirow[t]{2}{*}{ CDUS } & $264(66.0 \%)$ & $48(12.0 \%)$ & $38(9.5 \%)$ & $14(3.5 \%)$ & $36(9 \%)$ & 400 & \\
\hline & \multicolumn{5}{|c|}{ Tibial artery segments } & & \\
\hline CTA (100\%) & $288(72.0 \%)$ & $14(3.5 \%)$ & $22(5.5 \%)$ & $44(11 \%)$ & $36(9 \%)$ & 400 & \multirow[t]{2}{*}{$<0.001^{* *}$} \\
\hline CDUS (96\%) & 266 (66.7\%) & 38 (9.5\%) & 40 (10\%) & $34(8.5 \%)$ & $14(3.5 \%)$ & 400 & \\
\hline
\end{tabular}
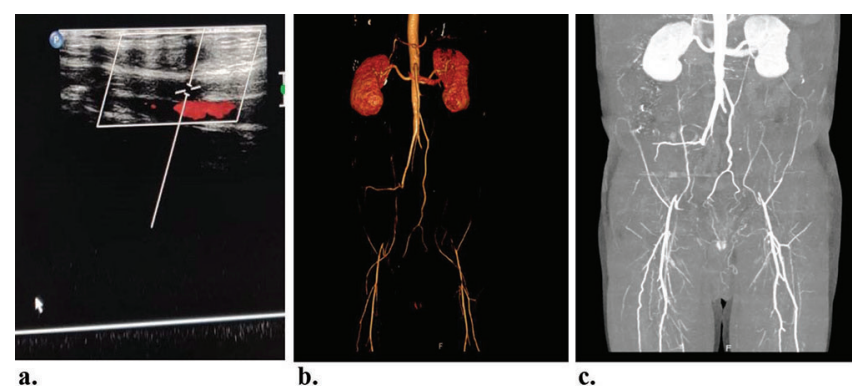

Case-1: CDUS (a) with spectral Doppler shows grade IV stenosis of left external iliac artery showing absent color uptake and absent waveform on spectral Doppler. CTA of Aortoiliac Segments - VR (b) and MIP (c) reconstructed coronal images showing Grade IV stenosis of left common iliac and bilateral external iliac arteries.

on CTA and out of 388 segments on CDUS 266 (66\%), 38 (9.5\%),40 (10\%), 34 (8.5\%),14 (3.5\%) segments on CDUS showed grade $0,1,2,3,4$ stenosis respectively. $4 \%$ segments could not be assessed on CDUS

Out of 400 vessel segments in femoropoliteal region 296 (74\%), 22 (5.5\%), 28(7\%), 16(4\%),38(9.5\%) segments on CTA and 264 (66\%), 48 (12\%), 38 (9.5\%), 14 (3.5\%), 36 (9\%) segments on CDUS showed grade $0,1,2,3,4$ stenosis respectively

Out of 1100 segments, 822 segments showed grade 0,64 segments showed grade 1, 60 segments showed grade 2, 72 segments showed grade $3 \& 86$ segments showed grade 4

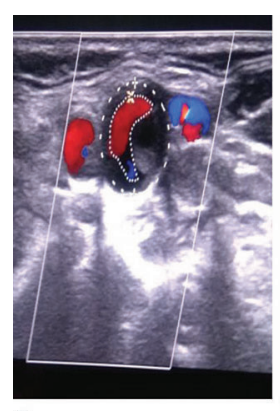

a.

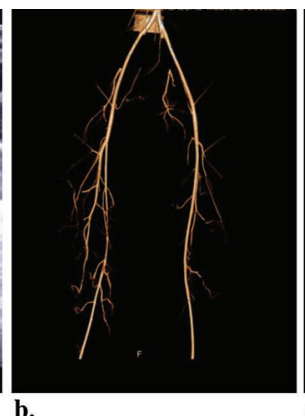

b.

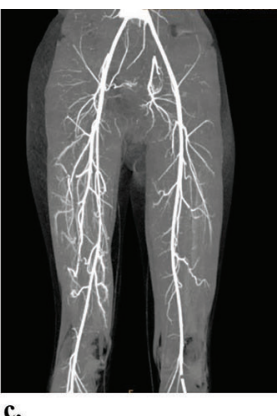

Case-2: CDUS (a) showing grade III stenosis of right superficial femoral artery. CTA of femoropopliteal segments -VR (b) and MIP (c) reconstructed coronal images showing Grade III stenosis of right superficial femoral artery.

stenosis on CTA and 748 segments showed grade 0,130 segments showed grade 1, 60 segments showed grade 2, 60 segments showed grade $3 \& 62$ segments showed grade 4 stenosis on CDUS (table-2,3).

Presence of collaterals on CTA was seen in $46 \%$ cases where as CDUS showed collaterals in only $26 \%$ cases. Out of 1100 vessel segments, $26 \%$ showed wall calcifications on CTA where as $12 \%$ showed wall calcification on CDUS.

\section{DISCUSSION}

Common risk factors for the PAD include increasing age, 
smoking, hypertension and diabetic mellitus. Out of the 100 patients we studied $72 \%$ were males and $28 \%$ were females indicating males are more affected by PVD compared to females which is in accordance with studies by Hughson et al. ${ }^{5}$ and Gareth Morris and his co-workers.

Our study concludes that distribution of disease increases in elderly population which is in accordance to study by Selvin E, Erlinger TP et $\mathrm{al}^{6}$ and Cossman et $\mathrm{al}^{7}$ where they found PAD prevalence increases dramatically with age with middle aged and the elderly patients are commonly affected.

We found $50 \%$ of smokers in our study population, out of which $65.5 \%$ showed hemodynamically significant stenosis in comparison with non smoking population in whom $24.2 \%$ showed significant stenosis with $\mathrm{P}$ value $<0.001$, showing significant correlation with smoking and stenosis. This is in accordance to study by Cole CW, et $\mathrm{al}^{8}$ where they found relative risk was 7 for ex-smokers and 16 for current smokers $(\mathrm{p}<0.001)$.

In our study $60 \%$ of patients were hypertensive, out of which $55.2 \%$ showed hemodynamically significant stenosis in comparison with non hypertensive population in whom 44.8\% showed significant stenosis with $\mathrm{P}$ value $~ 0.098$, showing correlation with hypertension and stenosis which is in accordance to study by Selvin E, Erlinger TP et al.

We found $60 \%$ of diabetic patients in our study population, out of which $79.3 \%$ showed hemodynamically significant stenosis in comparison with non diabetic population in whom 20.7\% showed significant stenosis with $\mathrm{P}$ value $<0.001$, showing significant correlation with diabetes mellitus and stenosis, which is in accordance to study by L. Norgren et $\mathrm{al},{ }^{9}$ where they found insulin resistance and diabetes was important risk factor which raised risk approximately 40 $50 \%$.

In our study the detection rate for the Grade 1 and 2 stenosis was higher for CDUS than CTA in all the three arterial regions. Detection rate for the Grade 3 stenosis was similar for both CDUS and CTA in the arterial segments of the aorto-iliac group. However CTA was better than CDUS for grade 3 stenosis in the arterial segments of femoro-popliteal and tibio-peroneal regions. Both CDUS and CTA were able to detect all the Grade 4 stenosis segments in the aortoiliac ( $\mathrm{P}$ value -0.277 ) and femoro-popliteal region ( $\mathrm{P}$ value -0.022).However greater number of the arterial segments with Grade 4 stenosis was detected by the CTA in the tibioperoneal region than CDUS with statistically significant $P$ value $<0.001$.

Thus our study showed though early stenotic lesions (Grade 1 and grade 2) were better picked by the CDUS in all the regions, Grade- 3 and 4 lesions were better demonstrated by the CTA in femoro popliteal \& tibio peroneal regions more significantly in the infrapopliteal region which correlated with study by Sathyabhuwan Singh et al. ${ }^{10}$ study who concluded that doppler sonography is better than CT angiography in the diagnosis of early onset (Grade I \& II) cases while $\mathrm{CT}$ angiography is more accurate in assessing grade- 3 and grade- 4 stenosis

In our study we found more number of diseased segments were detected by CDUS than CTA which correlates to the study by Kayhan $\mathrm{A}$ et $\mathrm{al}^{11}$ who found more diseased segments in CDUS than MDCT.

Collins $\mathrm{R}$ et al in $2007^{12}$ and Met $\mathrm{R}$ et al. in $2009^{13}$ also found CTA to be more accurate modality in assessing the presence and extent of peripheral arterial disease.

In this study, more number of vessel calcifications are detected by CTA than CDUS. Out of 1100 vessel segments assessed $26 \%$ showed vessel calcification on CTA in comparison with $12 \%$ detected on CDUS which is in accordance by Joshi A, Nimbkar A, Merchant S, et al in where they found CTA to be more accurate in detection of calcified plaque.

Among 100 cases, presence of collaterals were detected in 44 patients by CTA and in 26 patients in CDUS which concluded CTA was more efficient in detecting presence of collaterals when compared to CDUS which is in accordance by Rahul J. Shirol et $\mathrm{al}^{14}$ who concluded that CTA detected more collaterals than CDUS with statistically significant $p$ value.

We found that MDCT is more accurate in accessing multilevel stenosis, bilateral limb involvement \& length of stenosis.

\section{Limitations}

Limitation of our study is colour flow imaging and MDCTA findings were not compared with DSA, which is considered to be the gold standard technique in detecting lower extremity PAD. Therefore, results may underestimate the percentages of arteries with lesions that are actually detectable in patients with PAD.

\section{CONCLUSION}

We conclude that in suspected PAD patients, ultrasound is best screening tool.In assessing mild PAD patients who needs medical therapy and in whom the CT is contraindicated, ultrasound is the better imaging modality. In patients with moderate to severe PAD and in whom surgical intervention is required, CTA is preferred for thorough evaluation of entire lower limb arterial tree.

\section{REFERENCES}

1. Morgan R, Belli A M, Chun J Y. Peripheral vascular disease intervention. In: Adam A, Dixon A et al, editors. Grainger \& Allison's diagnostic radiology. 6th ed. Edenburgh: Churchill livingstone; 2015;1(4):2087.

2. Pollak AW, Norton P, Kramer CM. Multimodality imaging of lower extremity PAD: Current role and future directions. Circulation cardiovascular imaging. 2012;5(6):797-807.

3. Polak J F, Alessi-Chinetti J M. The peripheral arteries. Rumack M Carol editor Diagnostic ultrasound 4th ed.Phildelphia: Elsevier mobsy; 2011.p998

4. Joshi A, Nimbkar V, Merchant S, Mhashelkar Y, Talekar K. Role of CT angiography in the evaluation of peripheral vasculature using MSCT- our initial experience. Indian J Radio Imaging 2004; 14(1): 30915.

5. Hughson WG, Mann JI, Garrod E: Intermittent claudication: prevalence and risk factors. Br Med J 1978;1(2):1379-1381.

6. Selvin E, Hirsch AT. Contemporary risk factor control and walking dysfunction in individuals with peripheral 
arterial disease: NHANES 1999-2004. Atherosclerosis. 2008;201(2):425-433.

7. Cossman DV, Ellison JE et al. Comparison of contrast arteriography to arterial mapping with colour-flow duplex imaging in lower extremities. J Vasc Surg.1989; 10(5):522-529.

8. Cole CW, Hill GB, Farzad E, Bouchard A, Moher D, Rody K, et al. Cigarette smoking and peripheral arterial occlusive disease. Surgery. 1993;114(4):753-6.

9. L. Norgren, W.R. Hiatt, J.A. Dormandy, M.R. Nehler, K.A. Harris, F.G.R. FowkesInter-society consensus for the management of peripheral arterial disease (TASC II) Eur J Vasc Endovasc Surg, 33 (2007)

10. Sathyabhuwan Singh Netan, Rajesh Singh, Sanjay Kumar, Atees Singhal, Vishal Jain. CT Angiography Evaluation of Peripheral Vascular Disease and Comparison with Color Doppler Ultrasound. Journal of Evolution of Medical and Dental Sciences 2015;4(83): 14504-14514.

11. Kayhan A, Palabiyik F, Serinsoz S et al Multidetector Angiography versus arterial doppler USG in diagnosis of mild lower extremity peripheral diseases: Is a multidetector CT a valuable screening tool? Eur J Radiol 2012, 81(3):542-6.

12. Collins R, Cranny G, Burch J, et al. A systematic review of duplex ultrasound, magnetic resonance angiography and computed tomography angiography for the diagnosis and assessment of symptomatic, lower limb peripheral arterial disease. 2007

13. Met R, Bipat S, Legemate DA, Reekers JA, Koelemay MJ.Diagnostic performance of computed tomography angiography in peripheral arterial disease: a systematic review and meta-analysis. JAMA. 2009; 301(4):415-24.

14. Shirol R, Shetty A, T K C. Role of MDCT in Evaluation of Peripheral Vascular Disease of the Lower Limb Arteries and Comparison with Colour Doppler. Journal of Evolution of Medical and Dental Sciences 2015; 4(54): 9336-46

Source of Support: Nil; Conflict of Interest: None

Submitted: 23-01-2020; Accepted: 16-02-2020; Published online: 22-03-2020 